\section{Test Zone Size Characterization With Measured MIMO Throughput for Simulated MPAC Configurations in Conductive Setups}

\author{
Wei Fan ${ }^{(D)}$, Lassi Hentilä, Pekka Kyösti ${ }^{(D)}$, and Gert F. Pedersen
}

\begin{abstract}
This correspondence discusses over-the-air (OTA) testing for multiple-input multiple-output (MIMO) capable terminals, with an emphasis on test zone size characterization for multi-probe anechoic chamber (MPAC) OTA configurations. For an MPAC setup, it is important to understand the test zone size that can be supported by its design. Further, it is desirable that the test zone size should be determined in terms of measured throughput deviation. However, such works have not been reported in the literature yet, due to challenges in practical MPAC setups. In this correspondence, we propose to simulate the MPAC OTA configuration and MS design in a channel emulator and perform MIMO throughput measurements in a conductive setup. With the proposed scheme, we can investigate how large a test zone can be supported for the synthetic MPAC configuration in terms of throughput deviation. This scheme is attractive, since various MS antenna designs and MPAC configurations can be flexibly selected and test zone size in terms of measured MIMO throughput deviation can be achieved. Measurement results show that with a high antenna correlation at the base station (BS) side, measured throughput results would be low, irrelevant to MPAC OTA designs and antenna designs at the MS side. Therefore, the antenna correlation at BS side should be set uncorrelated to ensure that true MS performance can be measured. Further, spatial correlation accuracy at the MS side becomes critical for throughput accuracy only when correlation values are in the high region (e.g., $\rho>0.5)$.
\end{abstract}

Index Terms-Anechoic chambers, antenna measurements, fading channels, testing, radio propagation.

\section{INTRODUCTION}

Multiple-Input multiple-output (MIMO) technology is a key factor for achieving high data rate and good quality-of-service. There is a strong need for standard test methods to evaluate MIMO capable terminal performance [1]. MIMO over-the-air (OTA) testing is a promising solution, since the end-to-end performance of mobile terminals can be measured over-the-air, i.e. without the need to break the mobile terminal case [1]. Further, all critical aspects of the mobile terminal design (antenna, radio frequency (RF) front end, base-band algorithms, etc.) can be evaluated together. The wireless industry, through CTIA and 3GPP standardization bodies, has been working on different MIMO OTA candidate methods, where multi-probe anechoic chamber (MPAC) method has been selected [1].

This work was supported by the Innovation Fund Denmark via the Virtusuo project. The work of W. Fan was supported by the Danish council for independent research under Grant DFF611100525. The review of this paper was coordinated by Prof. T. Kuerner. (Corresponding author: Wei Fan.)

W. Fan and G. F. Pedersen are with the Antennas, Propagation and Radio Networking Section, Department of Electronic Systems, Aalborg University, Aalborg 9220, Denmark (e-mail: wfa@es.aau.dk; gfp@es.aau.dk).

L. Hentilä is with the Kesight Technologies Oy, Espoo 02600, Finland (email: lassi.hentila@keysight.com).

P. Kyösti is with Keysight Technologies Finland Oy, Oulu, Finland; and also with the Centre for Wireless Communications (CWC), University of Oulu, Oulu, FI-90014 Finland (e-mail: pekka.kyosti@keysight.com)

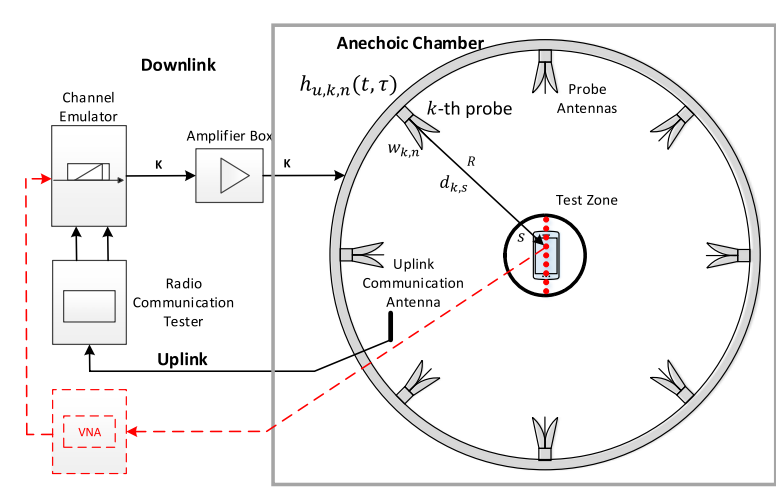

Fig. 1. An illustration of a MPAC setup. For terminal performance testing, a base station (BS) emulator and a real mobile station (MS) in test zone are used. For analysis of reconstructed channels in the test zone, a vector network analyzer (VNA) and a (virtual) array over test zone (denoted in red) are often used to replace the BS emulator and mobile terminal, respectively.

With the MPAC method, controllable RF multipath environments can be physically synthesized at the device under test (DUT) location in a repeatable way, by using a radio channel emulator connected to a circular array of probe antennas within an anechoic chamber, as shown in Fig. 1 [2]. A key question to be addressed in MPAC setups is how large a test zone can be supported for a MPAC configuration. The test zone is a geometrical zone located in the circular array center, where target channels can be accurately reproduced [2]-[4].

Extensive efforts have been taken to characterize the test zone size in the literature, where different figures of merit (FoMs) are proposed and analyzed for different channel emulation techniques. For the plane wave synthesis (PWS) technique, a field synthesis error $|E-\hat{E}|$ is used, where $E$ and $\hat{E}$ represent the target and emulated field, respectively [2], [3], [5]. For the prefaded signal synthesis (PFS) technique, the spatial correlation error $\left|\rho_{M S}-\hat{\rho}_{M S}\right|$ at the MS side is selected, where $\rho_{M S}$ and $\hat{\rho}_{M S}$ denote the spatial correlation under the target and emulated spatial channels, respectively [1], [2], [4]. For the spatial channel emulator method, channel emulation accuracy in terms of signal fading distributions and spatial correlation error are analyzed [6]. Various acceptable error thresholds have been proposed to determine the test zone size. For example, $\left|\rho_{M S}-\hat{\rho}_{M S}\right|<0.1$ was chosen in [1], [2], [4] and error vector magnitude (EVM) of less than $-15 \mathrm{~dB}$ was typically used for field synthesis error [2], [3], [5]. However, it has been difficult to define the acceptable error threshold in terms of field synthesis error or spatial correlation error, due to the fact that these error thresholds cannot be directly reflected in data rate deviation (in bits/s/Hz). An attempt in this direction is reported in [4], where channel capacity deviation $|C-\hat{C}|$ was utilized, with $C$ and $\hat{C}$ being the capacities of target and emulated channels, respectively. However, channel capacity only indicates the theoretical upper bound of the data rate, and cannot directly reflect practical MIMO terminal performance. Though these FoMs are needed to understand how accurate channel models are reproduced in the test zone at the MS side, their impact on system end-to-end performance metrics, e.g. data rate, is still unknown. Throughput has been selected as the final FoM in MIMO OTA standards to rank MIMO-capable terminals, as 
it reflects the end-user experience. Thus, it is highly desirable to use throughput deviation as a measure.

In the CTIA and 3GPP test plans, Reference Signal Recieved Power (RSRP) values required to reach the $70 \%, 90 \%$, and $95 \%$ of the theoretical maximum throughput of the test case should be recorded [1]. It would be desirable that we can quantify deviation in RSRP value (in $\mathrm{dB}$ ) at the specified throughput points measured under the target channels and under the emulated channels in MPAC setups.

Test zone size characterization in terms of throughput deviation in practical MPAC setups has not been reported in the literature, mainly due to practical problems, as detailed in Section II-A. It is desirable that we can characterize the test zone size in terms of RSRP deviation at certain throughput percentile. This is that most desirable metric in the MIMO OTA community for the MPAC design. However, this has not been solved so far and it is an open question in the literature. In this correspondence, an alternative solution is proposed, where the MPAC design and MS configuration can be analytically simulated in the channel emulator. With this method, the MS throughput performance under emulated channel models in the simulated MPAC configurations can be measured in the conductive setup. It is noted that this scheme is proposed to understand the test zone size for MPAC setups, which can facilitate the practical MPAC design.

The aim of this correspondence is twofold: to investigate whether test zone sizes determined with different FoMs based on their acceptable error levels in the literature have been valid and sufficient and to characterize test zone size for MPAC setups in terms of throughput deviation with the proposed scheme. To our best knowledge, these two aspects have not been addressed in the literature yet.

\section{METHOD}

\section{A. Problem Statement}

To investigate the relationship between test zone size and MPAC designs in terms of throughput deviation, different MS antenna spacings (i.e. within the supported test zone and outside the supported test zone) and different MPAC designs (i.e. number of probe antennas $K$ and radius $R$ ) are needed. As discussed earlier, it is still an open question how to characterize test zone size in terms of throughput accuracy in MPAC setups. The reasons are multi-fold:

1) MS antenna design (e.g. spacing, location and antenna characteristics) is fixed. To have various MS antenna spacings in test zone investigations, we need MS designs with various spacings. To ensure that throughput deviation is only caused by the MPAC design, not the DUT antenna characteristics, radiation patterns of antenna elements should be maintained the same for different spacings, which is not practical.

2) Practical MPAC design is typically fixed (i.e. number of probes antennas and radius).

3) Throughput under target channel models is required as a reference to determine the throughput accuracy in MPAC configurations. It is practically not feasible to measure throughput under target channel models in MPAC setups, as an infinite number of OTA antennas would be needed to reproduce the exact target channel models. In [7]-[9], a throughput prediction model based on receiver threshold power was proposed and validated in reverberation chambers for channel models with isotropic impinging power angle profiles. Promising though it is for non-isotropic channels as well, no throughput prediction model for standard spatial channel models are available yet.

It is noted that these challenges are not present with other FoMs. For example, the emulated field $\hat{E}$ over the test zone can be easily recorded with a calibration antenna, while target field $E$ can be analytically calculated [3], [5]. As for correlation analysis, $\hat{\rho}_{M S}$ can be obtained for various MS antenna spacings via a virtual array system (i.e. recording channel profiles at different array element positions with a single calibration antenna), due to full controllability of the reproduced channels in the MPAC setup [1], as shown in Fig. 1. Target spatial correlation $\rho_{M S}$ can be analytically obtained, based on the impinging power angle profiles of target models and MS array characteristics [4]. In the throughput measurements, antennas on the DUT are powered up and receiving signals simultaneously in the LTE downlink spatial multiplexing mode.

As explained earlier, unlike other FoMs which only focus on MS channel spatial characteristics, throughput is an end-to-end performance metric, which depends on BS antenna characteristics, spatial characteristics of the propagation channel and MS antenna characteristics.

\section{B. Throughput Accuracy Modeling}

As explained earlier, it is highly desirable that we can select various MS antenna spacings and MPAC designs for throughput accuracy analysis in MPAC setups. Since it is problematic for practical MPAC configurations, we propose to simulate the MPAC configuration and MS antennas in the channel emulator and perform throughput measurements in the conductive setups. Below, we first discuss the radio channel models (i.e. between the BS antenna ports and MS antenna ports) in the target and simulated MPAC configuration scenarios. After that, we explain the conductive setups to perform throughput measurements.

1) Target Channel Models: In this part, the well-defined geometry-based stochastic channel (GBSC) models are selected. Further, only vertically polarized channel models are considered for simplicity. Note that principle of the proposed method is general for throughput analysis for MPAC setups and can be directly applicable to other spatial channel models without limitations. For a MIMO system equipped with $U$ antennas at the BS side, and $S$ antennas at the MS side, the time-variant channel impulse response (CIR) for the $n$-th path (with $n \in[1, N]$ ) can be written as [2]:

$$
\begin{aligned}
h_{u, s, n}(t, \tau)= & \sqrt{p_{n}} \sum_{m} \sqrt{G_{B S}\left(\phi_{n, m}\right)} \sqrt{G_{M S}\left(\varphi_{n, m}\right)} \\
& \times \exp \left(j \frac{2 \pi}{\lambda}\left(\bar{\phi}_{n, m} \cdot \bar{r}_{u}\right)\right) \exp \left(j \frac{2 \pi}{\lambda}\left(\bar{\varphi}_{n, m} \cdot \bar{r}_{s}\right)\right) \\
& \times \exp \left(j 2 \pi \vartheta_{n, m} t+\Phi_{n, m}\right) \delta\left(\tau-\tau_{n}\right),
\end{aligned}
$$

where $p_{n}$ and $\tau_{n}$ denote the power and delay of the $n$-th path, respectively. $\lambda$ is the wavelength. $\delta($.$) is the Dirac function. G_{B S}$ and $G_{M S}$ are the antenna gain for the BS and the MS element, respectively. $\phi_{n, m}$, $\varphi_{n, m}, \vartheta_{n, m}, \Phi_{n, m}$ are the angle of departure, angle of arrival, Doppler frequency and initial phase of the $m$-th sub-path in the $n$-th path with $m \in[1, M] . \Phi_{n, m}$ is a random variable following uniform distribution in $[0,2 \pi] . \bar{\phi}_{n, m}$ and $\bar{\varphi}_{n, m}$ are unit direction vectors for angle $\phi_{n, m}$ and $\varphi_{n, m}$, respectively. $\bar{r}_{u}$ and $\bar{r}_{s}$ denote local vectors of antenna element $u$ in the BS array and $s$ in the MS array, respectively. 
2) Emulated Channel Models: The PFS technique is widely adopted as the channel emulation technique in MPAC setups, since it is capable of emulating all dimensions of the GBSC models [2]. With the PFS technique, CIRs radiated from the $k$-th probe antenna can be expressed as [2], [4]:

$$
\begin{aligned}
h_{u, k, n}^{O T A}(t, \tau)= & \sqrt{p_{n}} \sum_{m} \sqrt{G_{B S}\left(\phi_{n, m}\right)} \exp \left(j \frac{2 \pi}{\lambda}\left(\phi_{n, m} \cdot \bar{r}_{u}\right)\right) \\
& \times \exp \left(j 2 \pi \vartheta_{n, m} t+\Phi_{n, m, k}\right) \delta\left(\tau-\tau_{n}\right) \sqrt{w_{k, n}}
\end{aligned}
$$

where $w_{k, n}$ denotes the power weight for the $k$-th probe in the $n$-th path, which can be obtained via optimization algorithms [2], [4]. Note that we have $K$ different realizations for $\Phi_{n, m, k}$ with $k \in K$. This is needed to ensure that each probe antenna contributing to the same path has independent fading coefficient sequence with identical statistics [2].

To address complexities of modeling throughput in the practical MPAC setup (i.e. fixed MPAC configuration and MS antenna separation), we can reconstruct the received signals at the MS analytically, based on (2), as:

$$
\hat{h}_{u, s, n}(t, \tau)=\sum_{k=1}^{K} \sqrt{G_{M S}\left(\varphi_{k}\right)} \times \alpha_{k, s} \times h_{u, k, n}^{O T A}(t, \tau),
$$

where $\varphi_{k}$ denotes the angular location for the $k$-th probe. $\alpha_{k, n}$ is the propagation coefficient from the $k$-th probe antenna to the $s$-th antenna element in the MS, which is:

$$
\alpha_{k, s}=L\left(d_{k, s}\right) \times \exp \left(j \frac{2 \pi}{\lambda} d_{k, s}\right),
$$

where $L(\cdot)$ is the path-loss term and $d_{k, s}$ is the distance from the $k$-th probe to the $s$-th antenna element in the MS. Since the DUT size is much smaller than $R$, the probe antenna pattern is assumed constant over the angle region illuminating the DUT, and therefore ignored in (3).

3) Throughput Modeling: As a summary, (1) and (3) describes the radio channel models (i.e. including the effects of antenna characteristics at the BS and MS side, and the propagation environments) for the target scenario and emulated scenario in the simulated MPAC configurations. As explained earlier, to determine test zone size in terms of throughput accuracy, it is desirable that various MPAC configurations (i.e. with various available number of probe antennas and physical dimensions) and various MS antenna spacings should be flexibly selected, with all MS antennas active and powered up. This can be easily realized, since arbitrary settings can be selected for MS antenna spacings and MPAC configurations in (3). Once the radio channel models for the target case and emulated case in simulated MPAC scenarios are calculated, the throughput can be measured in a conductive setup for each case, as shown in Fig. 2 (top). The measurement system consists of a BS emulator, a channel emulator and a MS. The MS can be placed in a small RF shielded box to eliminate external interference and noise, as shown in Fig. 2 (below). This solution is attractive, since various MS antenna spacings and MPAC configurations can be set as parameters in (3). Further, throughput for the target model can be directly measured in the conducted setup and utilized as a reference. Therefore, problems for test zone characterization with throughput accuracy discussed for practical MPAC setups in Section II, i.e. various MS spacings, MPAC configurations, throughput under target channel models, can be addressed with the proposed scheme.
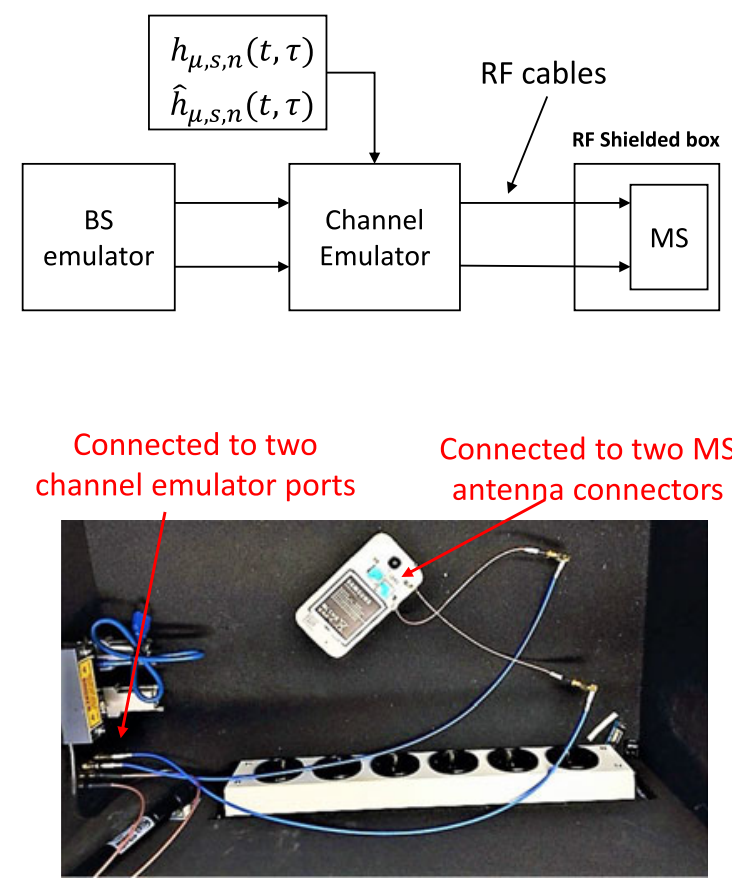

Fig. 2. Illustration of the system for throughput accuracy modeling (top) and a photo of the conducted measurement in the RF shielded box (below). $h_{u, s, n}(t, \tau)$ denoted the target CIRs and $\hat{h}_{u, s, n}(t, \tau)$ represents the emulated CIRs in the simulated MPAC configuration between the BS antenna ports and MS antenna ports, respectively.

It is noted that antennas at the BS/MS side and the propagation channel are modeled separately in (1) and (3) for the target channel models and emulated channel models in the simulated MPAC configurations. In typical conductive setups, MS antennas are detached from antenna ports on the mobile terminal, while radio frequency (RF) cables are used to guide the signals to the antenna ports directly. As a result, the terminal case needs to be opened. In [10], a wireless cable method was proposed to replace cable conductive testing.

\section{MEASUREMENT SETUP}

A photo of the measured MS in an RF shielded box is shown in Fig. 2 (below). The measurement system is explained in Table I. The throughput measurement procedure is detailed in [1]. Note that the interference and noise are not modeled in the measurement. We first adjusted the attenuators in the channel emulator to achieve RSRP values required for maximum throughput. Then for each throughput measurement point, RSRP values were decreased with $1 \mathrm{~dB}$ step until the LTE throughput reaches below $10 \%$ of the maximum throughput. For each throughput measurement point, 20000 sub-frames per stream were utilized, as suggested in [1]. A total of 24 throughput measurements were performed (i.e. 6 different MS antenna spacings for two SCME channels and for both target and reconstructed models). Note for easy exhibition, we use the throughput percent, defined as the throughput normalized by its maximum value (20.6 Mbit/s) [7], [8].

\section{Measurement Result Analysis}

\section{A. Spatial Correlation Analysis}

The spatial correlation can be calculated based on the target and reconstructed CIRs, as detailed in [4]. The spatial correlation values 
TABLE I

SETUP AND SPECIFICATIONS OF EACH COMPONENT IN THE MEASUREMENT SYSTEM

\begin{tabular}{ll}
\hline \hline Component & \multicolumn{1}{c}{ Setup and specifications } \\
\hline BS emulator & - Model: Anritsu MT8820C \\
& - Modulation and coding scheme (MCS) index: 13 \\
& - LTEme structure: frequency division duplex (FDD) \\
& 1842.5 MHz; Uplink frequency: $1747.5 \mathrm{MHz}$ \\
& - Channel bandwidth: $10 \mathrm{MHz}$ \\
& - LTE mode: $2 \times 2$ open loop spatial multiplexing \\
& - Model: Keysight Propsim F32 \\
Radio channel & BS array: Two vertically polarized isotropic antenna \\
emulator & elements with $10 \lambda$ spacing. \\
& - Channel models: Target and reproduced vertically \\
& polarized SCME Urban micro (UMi) and Urban macro \\
& (UMa) channel models. \\
& - OTA configuration: A uniform MPAC setup with eight \\
& vertically polarized probe antennas \\
& - MS array: Two vertically polarized omnidirectional \\
& antenna elements with $0.5 \lambda, 0.7 \lambda, 0.9 \lambda, 1.1 \lambda, 1.3 \lambda$, \\
& $1.5 \lambda$ antenna spacing, respectively \\
& - Model: Samsung Galaxy S4
\end{tabular}

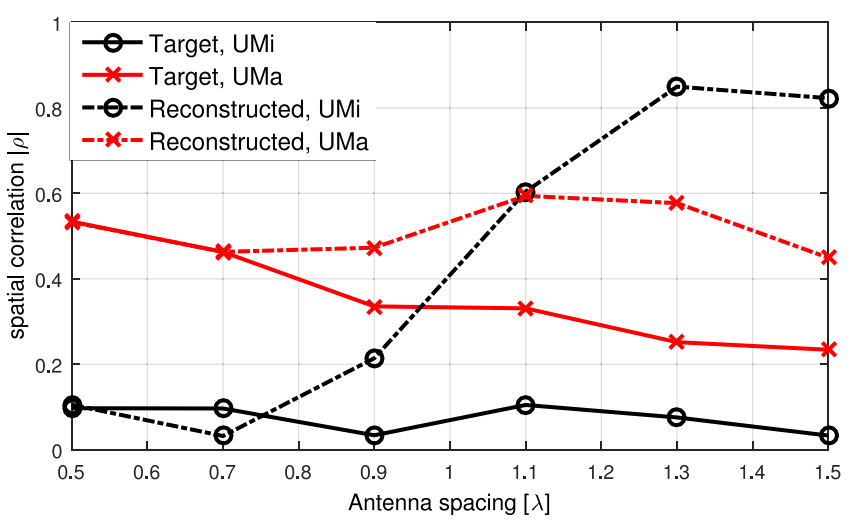

Fig. 3. Spatial correlation at the MS side for the target and reconstructed channel models with various MS antenna spacing.

at the BS side are 0.01 and 0.9 for the SCME UMi and UMa channel model, respectively. Spatial correlation values of reconstructed channels match perfectly with target ones at the BS side, as expected. Spatial correlation values at the MS side for target and reconstructed channel models are shown in Fig. 3. As we can see, a good agreement between target and emulated curves up to $0.7 \lambda$ is observed for both channels. When antenna separation exceeds $0.7 \lambda$, the emulated spatial correlation will be larger than the target for both channel models, as a result of limited number of probes. As discussed earlier, the spatial correlation accuracy at MS side $\left|\rho_{M S}-\hat{\rho}_{M S}\right|$ is dominantly used in the literature to determine test zone size.

\section{B. Throughput Analysis}

1) UMi Channel Model: The measured throughput for MS arrays with various spacings under target and reconstructed UMi channel models are shown in Fig. 4. Throughput curves of target channels with different MS antenna spacings are quite similar, with a difference in RSRP values less than $0.1 \mathrm{~dB}$ at $70 \%, 90 \%$ and $95 \%$ throughput, as

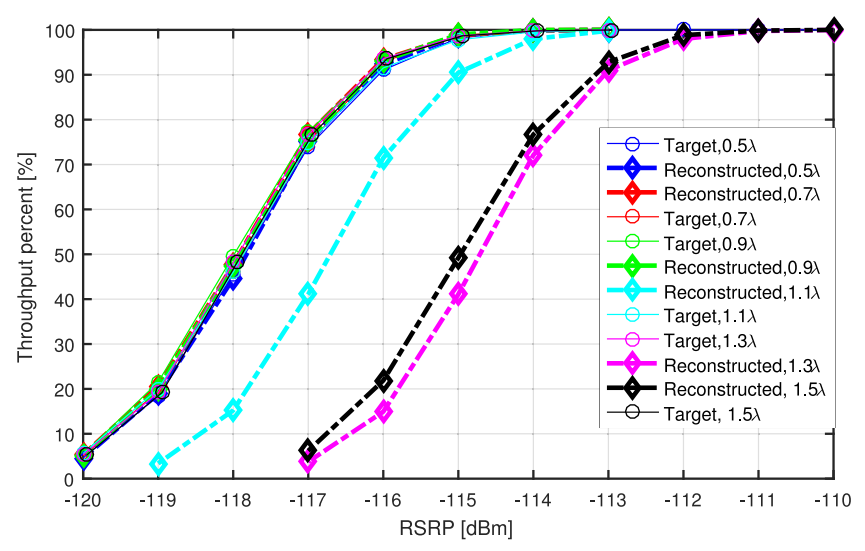

Fig. 4. Measured throughput versus RSRP values for target and reconstructed SCME UMi channel models with various MS antenna spacing.

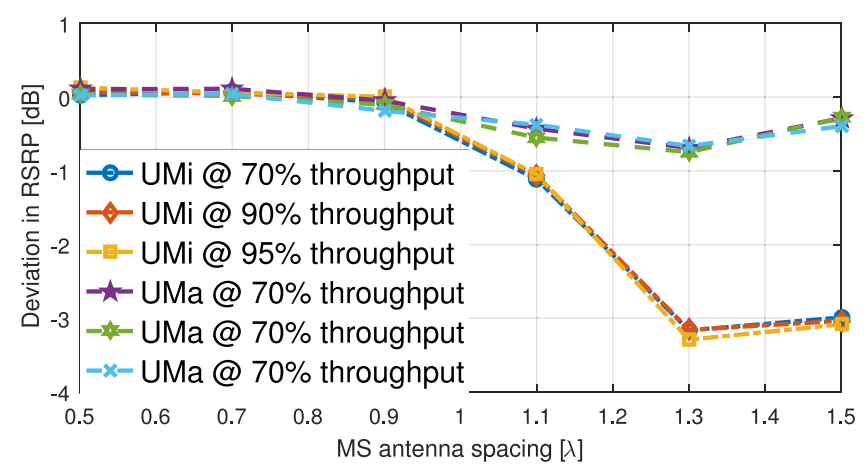

Fig. 5. Deviations in RSRP values at 70\%, $90 \%$ and $95 \%$ throughput for the target and reconstructed SCME UMi and UMa channel models.

shown in Fig. 4. This is because spatial correlation values at the MS side are equally low (i.e. around 0.1 shown in Fig. 3) for the target SCME UMi models with different MS antenna spacings. An excellent agreement between measured throughput under target and emulated channels is achieved for MS antenna spacing up to $0.9 \lambda$, with deviations in RSRP less than $0.1 \mathrm{~dB}$ at 70\%, $90 \%$ and $95 \%$ throughput, as shown in Fig. 5. With MS antenna separation $0.9 \lambda$, though $\left|\rho_{M S}-\hat{\rho}_{M S}\right|$ is around 0.2 , throughput deviation is negligible, since both target and emulated spatial correlation are in the low region (i.e. below 0.5), as shown in Fig. 3. With MS antenna separation larger than $0.9 \lambda$, throughput deviation gets larger, since emulated spatial correlations are in the high region, while target ones are in the low region. Deviations in RSRP values around $1 \mathrm{~dB}, 3 \mathrm{~dB}$ and $3 \mathrm{~dB}$ are observed at $70 \%, 90 \%$ and $95 \%$ throughput for MS spacing $1.1 \lambda, 1.3 \lambda$ and $1.5 \lambda$, respectively.

With a low correlation at the BS side (e.g. 0.01 for the SCME UMi channel model), the measured throughput result is highly sensitive to the correlation value at the MS side when it is in the high region. The antenna correlation at the MS side depends on its antenna design. Therefore, performance of MS antennas can be reflected in the throughput results.

2) UMa Channel Model: The measured throughput results for the UMa channel models are shown in Fig. 6. The measured throughput results for target UMa models are generally much lower than for target UMi models, with a difference up to $4 \mathrm{~dB}$ in RSRP in $70 \%$, $90 \%$ and $95 \%$ throughput throughput values. This is due to the fact that 


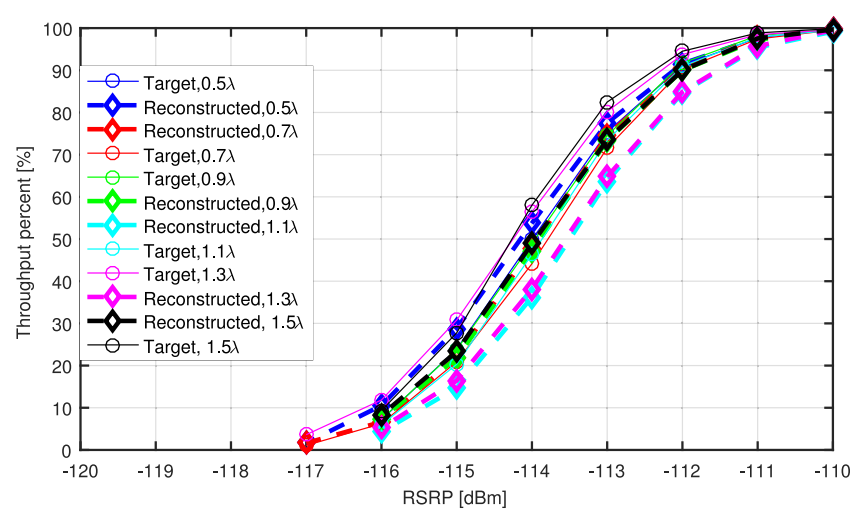

Fig. 6. Measured throughput versus RSRP values for target and reconstructed SCME UMa channel models with various MS antenna spacing.

UMa channel models are ill-conditioned with a very high BS correlation (i.e. 0.9). Throughput curves of target channels with different MS antenna spacings are quite similar, with a difference in RSRP values up to $0.5 \mathrm{~dB}$ at $70 \%, 90 \%$ and $95 \%$ throughput, as shown in Fig. 6. Further, deviations in RSRP values at 70\%, 90\% and 95\% throughput for different MS antenna spacings are quite small, with values only up to $0.7 \mathrm{~dB}$, as shown in Fig. 5. The smaller deviation for the UMa channel model, compared to for the UMi channel model, is due to the fact that spatial correlations at the BS side are high, which means the measured throughput under target and emulated channels are equally low. The impact of $\left|\rho_{M S}-\hat{\rho}_{M S}\right|$ on throughput deviation becomes negligible.

3) Discussion: To allow MS performance to be truly measured, BS antenna correlation should be set with a low correlation. With a low correlated BS antenna array, correlation deviation at the MS side $\left|\rho_{M S}-\hat{\rho}_{M S}\right|$ is not very critical for throughput accuracy if both $\rho_{M S}$ and $\hat{\rho}_{M S}$ are in low correlation region. However, throughput accuracy becomes highly sensitive to $\left|\rho_{M S}-\hat{\rho}_{M S}\right|$, when the $\hat{\rho}_{M S}$ or $\rho_{M S}$ are in the high region. If the acceptable error level for throughput deviation in the MPAC configuration is selected to be $0.5 \mathrm{~dB}$ in RSRP value at the $70 \%$ throughput values, the determined test zone is $0.9 \lambda$ and at least $1.5 \lambda$ for the SCME UMi channel and SCME UMa channel, respectively. In practical MPAC design, the determined test zone size should be applicable for arbitrary channel models. Therefore, it is suggested that the test zone size in term of measured throughput accuracy should be determined with channel models with low correlations at the BS side.

\section{CONCLUSION}

One of the key questions to be addressed when designing a MPAC setup is how large test zone can be supported with a limited number of probes and physical setup dimension. The current work proposes a conductive setup, where MPAC configurations and MS antennas are simulated in the channel emulator, to investigate the test zone size in terms of throughput deviation. This scheme is attractive for MPAC designs, since various MS antenna designs and MPAC configurations can be flexibly selected in the channel emulator and test zone size in terms of measured MIMO throughput deviation can be achieved. With the proposed scheme, we aim to firstly investigate whether test zone sizes determined with different FoMs based on their acceptable error levels in the literature have been valid and sufficient to characterize test zone size for MPAC setups in terms of throughput deviation.

Two measurements were conducted with the SCME UMi and UMa models. Measurement results in the UMi channel models show that at $70 \%, 90 \%$ and $95 \%$ throughput, an excellent agreement between measured throughput under target and emulated channels is achieved for MS antenna spacing up to $0.9 \lambda$, with deviations in RSRP less than $0.1 \mathrm{~dB}$, while deviations in RSRP values around $1 \mathrm{~dB}, 3 \mathrm{~dB}$ and $3 \mathrm{~dB}$ are observed for MS spacing $1.1 \lambda, 1.3 \lambda$ and $1.5 \lambda$, respectively. Further, measurement results demonstrate that BS effects should be removed, via ensuring a low BS correlation to measure the true performance of different MS designs. Further, correlation error, which is typically adopted in the literature to determine test zone size, only proves to be highly relevant for throughput accuracy modeling when the correlation values are in high region (e.g. $\rho>0.5$ ). Moreover, it is suggested that the test zone size for MPAC setups in term of measured throughput accuracy should be determined with channel models with low correlations at the BS side.

The proposed scheme can be utilized to determine test zone size in terms of throughput deviation of arbitrary channel models and DUT antenna designs in MPAC configurations. In the correspondence, we selected vertically polarized channel models, and MSs with two vertically polarized omni-directional antenna elements of various antenna spacings as examples to demonstrate the idea. In the future work, the impact of different MS designs and channel models on the test zone size in terms of throughput deviation should be investigated. It would be desirable to achieve a function mapping the DUT size and the probe configuration to the deviation in RSRP in $\mathrm{dB}$ units at given throughput percentile. To determine this, future work should apply the proposed method in the paper for many different DUT types and sizes with many different probe configurations.

\section{REFERENCES}

[1] "Test plan for $2 \times 2$ Downlink MIMO and transmit diversity over-theair performance," CTIA Certification, Tech. Rep., Version 1.1, Aug. 2016.

[2] P. Kyösti, T. Jämsä, and J.-P. Nuutinen, "Channel modelling for multiprobe over-the-air MIMO testing," Int. J. Antennas Propagat., vol. 2012, 2012, Art. no. 615954, doi:10.1155/2012/615954.

[3] C. Schirmer et al., "3d wave-field synthesis for testing of radio devices," in Proc. 8th Eur. Conf. Antennas Propag., Apr. 2014, pp. 3394-3398.

[4] W. Fan, P. Kyösti, J. O. Nielsen, and G. F. Pedersen, "Wideband MIMO channel capacity analysis in multiprobe anechoic chamber setups," IEEE Trans. Veh. Technol., vol. 65, no. 5, pp. 2861-2871, May 2016.

[5] J. T. Toivanen, T. A. Laitinen, V. M. Kolmonen, and P. Vainikainen, "Reproduction of arbitrary multipath environments in laboratory conditions," IEEE Trans. Instrum. Meas., vol. 60, no. 1, pp. 275-281, Jan. 2011.

[6] K. Sakaguchi, T. Sakata, and A. Yamamoto, "Performance evaluation of spatial correlation characteristics for handset antennas using spatial fading emulator based on Clarke's model," IEICE Trans. Commun., vol. 93, no. 10, pp. 2514-2522, 2010.

[7] A. Hussain, B. P. Einarsson, and P. S. Kildal, "Mimo ota testing of communication system using SDRS in reverberation chamber [measurements corner]," IEEE Antennas Propag. Mag., vol. 57, no. 2, pp. 44-53, Apr. 2015.

[8] X. Chen, "Throughput modeling and measurement in an isotropicscattering reverberation chamber," IEEE Trans. Antennas Propag., vol. 62, no. 4, pp. 2130-2139, Apr. 2014

[9] X. Chen, "Experimental investigation and modeling of the throughput of a $2 \times 2$ closed-loop mimo system in a reverberation chamber," IEEE Trans. Antennas Propag., vol. 62, no. 9, pp. 4832-4835, Sep. 2014.

[10] W. Yu, Y. Qi, K. Liu, Y. Xu, and J. Fan, "Radiated two-stage method for LTE MIMO user equipment performance evaluation," IEEE Trans. Electromagn. Compat., vol. 56, no. 6, pp. 1691-1696, Dec. 2014. 